\title{
Dermal Microfilariasis and Leprosy
}

\author{
A. C. McDOUGALL \\ The Slade Hospital, Headington, Oxford OX3 7JH, U.K. \\ and \\ H. WAUDBY \\ The Leprosy Study Centre, 57 a Wimpole Street, \\ London WIM $7 D F$, U.K.
}

\begin{abstract}
In the course of studying over 13,000 biopsies referred to the Leprosy Study Centre in London between 1952 and 1976, it was found that approximately 26\% showed no evidence of leprosy on histopathological examination. Some of these were normal and others showed minimal non-specific changes, but many revealed a wide range of dermatological and tropical conditions, amongst which the most important and frequently recurring was microfilariasis, due predominantly to Onchocerca volvulus, but also including infections with Dipetalonema streptocerca. Biopsies were submitted from Zaire, Nigeria, Sierra Leone and Cameroon, mostly on account of a suspicion of leprosy, or in order to confirm a diagnosis of leprosy, in some cases after treatment had been started with dapsone.

A 12-year period (1964-1976) has been selected for detailed study and the histopathological findings are considered in close relation to the doctor's letter or clinical information supplied. It is apparent that in geographical areas where both leprosy and onchocerciasis or streptocerciasis are endemic, there is continuing confusion-even amongst experienced observers-which may lead to errors in the diagnosis, classification, assessment and follow-up of patients with leprosy.

Skin biopsies, with appropriate attention to (1) the body site selected, (2) laboratory technique, and (3) the careful examination of serial sections, may be invaluable in minimizing or eliminating these errors.
\end{abstract}

\section{Introduction}

In the case of both leprosy and onchocerciasis, it has not infrequently been stated that the diagnosis "should present no difficulty to the experienced observer". But in practice, patients suffering from these diseases may be handled by a succession of doctors or auxiliaries who have limited experience of the clinical appearances of the 2 conditions or of the laboratory procedures needed for accurate differentiation. In the case of leprosy, diagnostic confusion is particularly likely to arise in (1) the early stages of lepromatous disease, (2) the "doubtful macule", including those patients classified as "indeterminate", and (3) borderline (dimorphous) and tuberculoid cases after varying periods of treatment.

As a nucleus for teaching and a stimulus to research and histopathological 
diagnosis, the Leprosy Study Centre in London was conceived in the early 1950's by Dr R. G. Cochrane and by mid-1976 had handled over 13,000 biopsies from various parts of the world, often from doctors working in areas where leprosy is prevalent but who have no facilities for the processing and interpretation of biopsies.

In the present study we have concentrated on the 12 years from 1964 to 1976 , since, during this period techniques of fixation, staining and interpretation were uniform and also because it contained a considerable number of skin biopsies in which microfilariae were present, either alone, or with evidence of leprosy. These have been examined in close relation to the doctor's letter or clinical information supplied. The present paper analyses the results with particular attention to the extent to which onchocerciasis or streptocerciasis may confuse the diagnosis and management of leprosy.

\section{Patients and Methods}

The patients came from the south west region of Cameroon, Nigeria, Sierra Leone and the north west and north east regions of Zaire.

submitted to London because of a clinical suspicion of leprosy, or uncertairit in diagnosis, or to confirm the diagnosis of leprosy, in some instances in patients already under treatment with dapsone. One patient only was recorded as having recently had treatment with diethylcarbamazine (Banocide). With rare exceptions, fixation was in formol-Zenker, with transfer to 76\% alcoliol 15-24 h later, and staining was a combined trichrome with the Fite-Faruso modification of Ziehl-Neelsen; "TRIFF" (Wheeler, Hamilton and Harman, 196\%) Tissuss were mounted in paraffin and cut at $5 \mu \mathrm{m}$, at least 6 , and often inany more serial sections being examined, the TRIFF technique revealing bacilli, infiltrating cells and microfilariae (MF) with equal clarity (Figs 1-4). The criteria for the classification of leprosy in sections were essentially those of the 5-group system (Ridley and Jopling, 1966).

In this study, we have not attempted a compl a different

volvulus (OV) from Dipetalonema streptocerca tyS) except in. a group of 69 patients from one area of Zaire, in which DS wc.. vlearly identified, using the diagnostic criteria listed by WHO (Buck, 1974). Heads and tails were however, not invariably encountered in sections and more reliance was placed on the measurement of body width, using a screw-micrometer eyepiece and reference slide $(1 \mathrm{~mm}-1000 \mu \mathrm{m})$.

\section{Results}

During the 12 years of this study, J bropsies were submitted to the centre, of which 1156 were from tissues wiher than skin (mainly peripheral nerve), leaving a total of 6924 skin biopsies. From this total, 314 were pusitive tor MF, 125 (39.8\%) showing MF only, ano the remaining 189 (60.2\%) MF with leprosy.

(a) BIOPSIES SHOWING MF ONLY

Clinical findings

Lesions were variable in size and distribution but most often recorded on the trunk. They were all macular and most had vague or irregular edges though a few were described as distinct or sharp; many were coalescing. Two patients had 
hyperpigmented lesions but the vast majority were hypopigmented, though occasionally with normal or even increased pigmentation in the centre. Referring letters noted some degree of anaesthesia in approximately $20 \%$ of all patients in this group. Itching was a virtually constant symptom.

Reasons for submission of biopsy

These may be summarized as follows:

\begin{tabular}{|c|c|c|}
\hline & Number & $\%$ \\
\hline 1. ?Diagnosis* & 12 & 9.6 \\
\hline 2. & 68 & 54.5 \\
\hline 3. & 14 & 11.2 \\
\hline $\begin{array}{l}\text { 4. Leprosy; for confirmation of } \\
\text { diagnosis for classification }\end{array}$ & 31 & 24.8 \\
\hline Total & 125 & \\
\hline
\end{tabular}

* Includes 3 patients: ?mycosis.

\section{Histopathology}

MF were found at various depths in the dermis, but most commonly high up, often close to the basal layer of epidermis and ascending into papillae between rete pegs. They were found only rarely within the epidermis and not recorded in surface keratin; intra-epidermal abscesses were not observed. They frequently lay between collagen fibres without exciting any cellular reaction, but were also seen with an infiltrate vhich was characteristically in the upper layers and mainly histiocytic, though with variable numbers of lymphocytes, mast cells and eosinophils. Plasma cells, however, were the dominant cell type in many sections, and their presence around compietely normal appendages (Fig. 1) was an indicator of microfilarial pathology in several instances. Many biopsies showed MF lying in the lumen of capillaries in transverse or longitudinal section.

\section{Microfilarial species identification}

As already stated, this was not attempted in all the biopsies available in this study. However, in 69 biopsies from one area of Zaire, approximately $4 \mu \mathrm{m}$ were recorded, and taken together with the available epidemiological, clinical and skin-snip data, these have been interpreted as indicative of DS. The vast majority, for similar reasons, and in whom a sample survey of biopsies gave average widths of $7 \mu \mathrm{m}$ or more, have been interpreted as OV.

\section{(b) BIOPSIES SHOWING MF WIT H LEPROSY}

\section{Clinical findings}

The clinical picture recorded was essentially that of the type of leprosy as classified on histopathological examination, viz.

Lepromatous 34 (18\%)

Borderline (dimorphous) 137 (72.4\%)

Tuberculoid $7(3.6 \%)$

Indeterminate $3(1.6 \%)$

In the remaining 8 biopsies $(4.2 \%)$ there were tissue and cellular changes strongly suggestive or indicative of leprosy, but insufficient for exact classifica- 


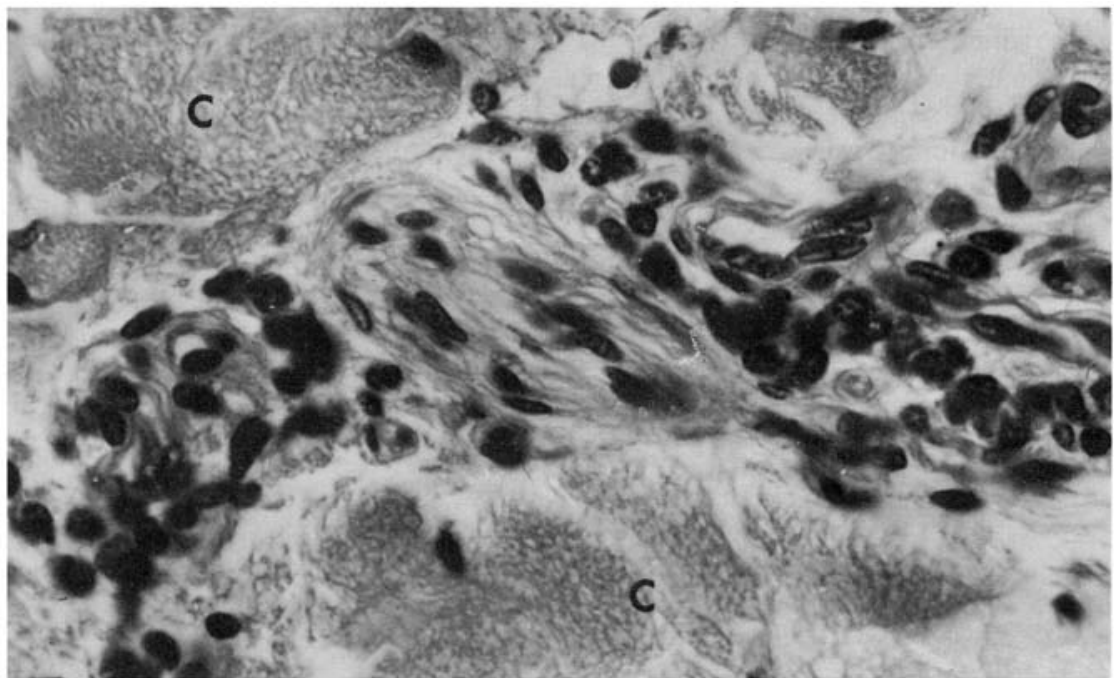

Fig. 1. Micro filariasis only. A completely normal nerve (centre) in the upper dermis is flanked by an infiltrate in which plasma cells are dominant. This is uncharacteristic of any form of leprosy and in fact MF were found in a closely adjacent field. C, collagen. TRIFF. Original magnification $\times 1250$.

tion. Besides the classical features expected with the types of leprosy recorded above, one sign, namely the hypopigmented macule (or macules), and one symptom, namely itching, were recorded in well over half the cases.

Apart from the biopsies described in this study, a further group from areas where OV or DS are prevalent and which were originally regarded as indeterminate leprosy* are currently being re-examined and assessed in serial sections. The results to date show that in a considerable number the clinical and histopathological features originally suggesting leprosy were in fact due exclusively to changes consequent on the presence of MF.

Reasons for submission of biopsy

These may be summarized as follows:

\begin{tabular}{lrr}
\hline & Number & $\%$ \\
\hline 1. ?Diagnosis* & 2 & 1 \\
2. ?Leprosy & 33 & 17.5 \\
3. ?Leprosy, ?filariasis & 8 & 4.2 \\
4. Leprosy; $\quad$ diagnosis or classification & 146 & 77.2 \\
$\quad$ Total & 189 & \\
\hline
\end{tabular}

* Includes 1 ?mycosis.

* WHO definition of Indeterminate leprosy:

bacteriologically pos ve, presenting flat skin lesions which may be hypopigmented or erythematous... the oup consists essentially of the "simple macular cases'" Leprosy Control, 19‘.j). 


\section{Histopathology}

MF lay as described in the cases above in which they were the only pathogen seen in sections, but were also frequently seen in or closely associated with the typical infiltrates of tuberculoid, borderline (dimorphous) (Figs 2 and 3) and lepromatous leprosy (Fig. 4). Even where numerous, there was no evidence to suggest that they had modified the patient's cellular response to the presence of the leprosy bacillus. Incontinence of pigment into the dermis or its presence in melanophores was common, but an examination of the basal layers of the epidermis gave no definite indication of depletion of melanoblasts or clear cells. As in the material showing MF only, this group also had numerous examples of MF lying in the lumen of capillaries. (More detailed histopathological findings of this dual pathology are to be described in a separate publication.)

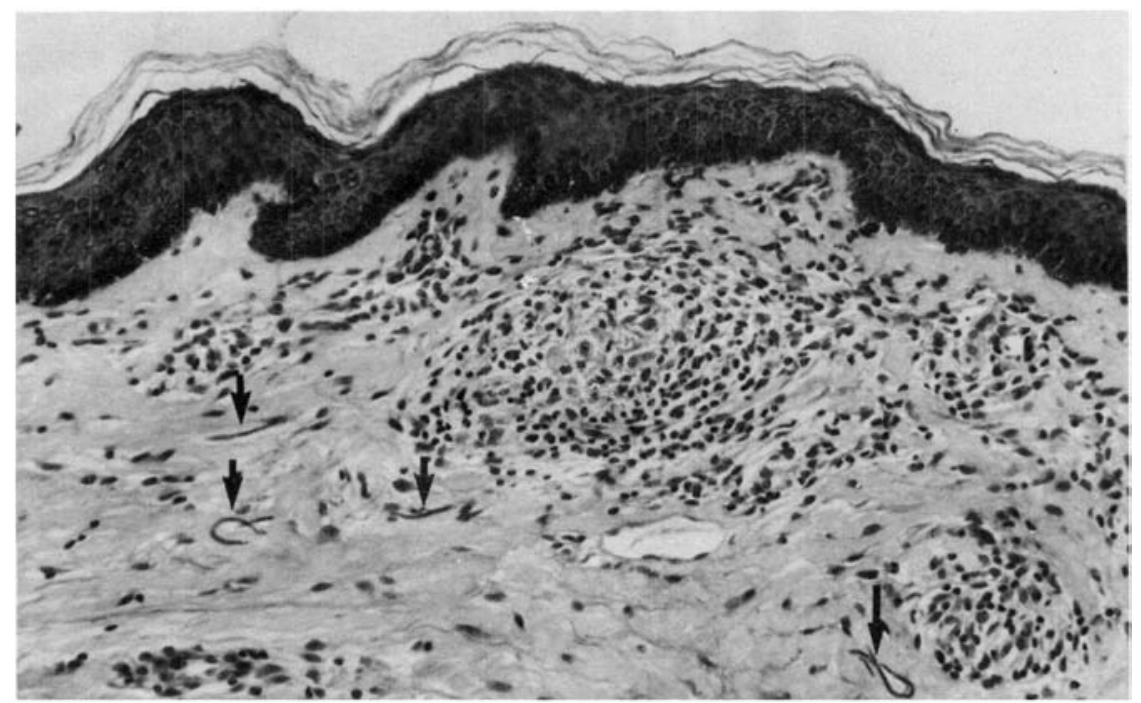

Fig.

surrounded by lymphocytes in the upper dermis and nerves lower down were similarly infiltrated. MF (arrowed) were numerous between these lesions. TRIFF. Original magnification $\times 500$.

\section{Discussion}

It is important to emphasize that the figures revealed in this analysis are to some extent fortuitous, depending upon the enthusiasm and interest of doctors or leprosy workers who were in touch with the centre during the period of study and they certainly do not represent the prevalence of the 2 diseases in the areas concerned. Furthermore the clinical notes accompanying each biopsy were not uniformly adequate, partly because some doctors working in filarial regions had long since regarded the entire population as being infected at some time or other. Nevertheless, a careful reading of the clinical information has indicated the reason for submission of the biopsy in all cases, revealing an area of confusion-and even frank error-which mainly affects the diagnosis and management of leprosy.

It was originally thought that the finding of MF in sections where leprosy was 


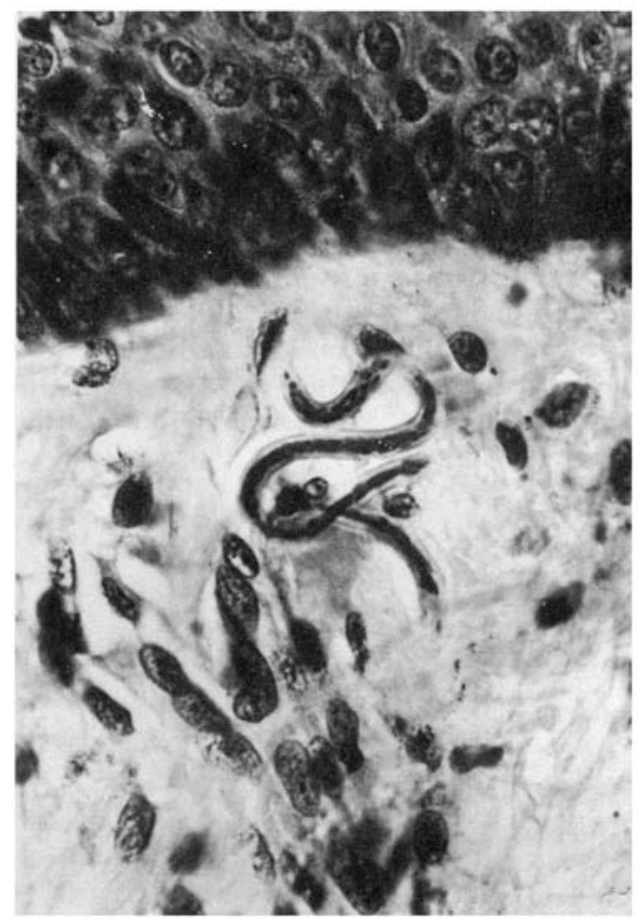

Fig.

and infiltrate. TRIFF. Original magnification $\times 1250$.

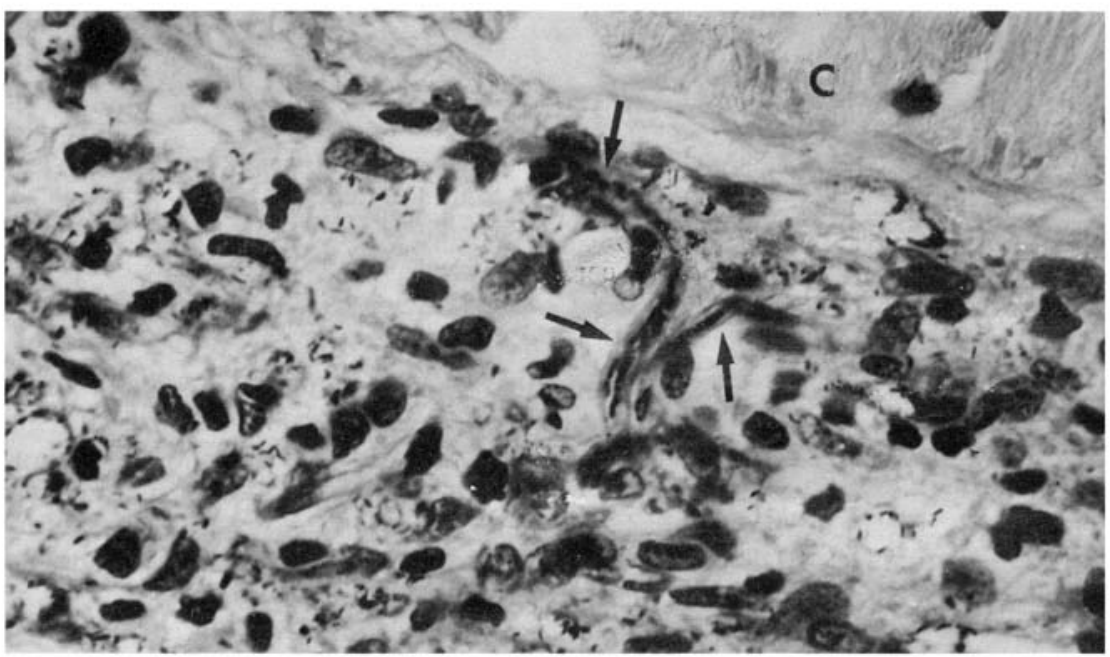

Fig.

lepromatous infiltrate consisting mainly of macrophages packed with Mycobacterium leprae. C, collagen. 
also present might be incidental and of little significance. However, this conclusion became untenable after an examination of the clinical notes and of a number of cases in which several biopsies has been submitted over a period of years. These showed that onchocercal changes such as hypopigmentation, chronic dermatitis, erythema, secondary surface infections, etc., may create at least 2 practical difficulties in the mind of the doctor dealing with a patient already proven to have leprosy: (1) it becomes difficult to assess clinical progress and reactional changes; new MF lesions, or the spreading of established ones, may create the impression that the patient's leprosy is not being held by treatment, and (2) the selection of a profitable site for biopsy may be rendered difficult. As a symptom, itching is misleading, for in leprosy it is not characteristic but may however be present and can be confused in local tribal languages with words intended to mean (medically) paraesthesiae. As a sign, loss of sensation is of paramount importance in the diagnosis of leprosy in the field, but may be absent at certain stages of the disease and is liable to misinterpretation unless meticulously elicited. Perhaps even more misleadingly, as this study shows, it may be present in some patients who have MF but not leprosy, almost certainly due to the presence of chronic epidermal and dermal pathology, and to difficulties of interpretation.

The very real confusion encountered in the diagnosis of these diseases in the field is still more apparent from the 125 biopsies in which there was no evidence of leprosy of any kind, but in which MF were readily found. Some of these patients had been on dapsone for a year or more and the combined clinical and histopathological data of this study strongly suggest that leprosy had been incorrectly diagnosed. In others, however, the clinical information pointed to onchocerris or streptocerciasis as the correct (and only) diagnosis, but in reporting inese "leprosy-negative" biopsies through the years, care has been taken to emphasize that the absence of any changes due to leprosy referred only to the biopsy submitted at that time. Indeed in some instances (where the clinical notes suggested leprosy but the biopsy was negative), a request for another biopsy from a more likely skin site has produced positive histopathology. Such cases confirm that oncho- or streptocerciasis can interfere with the selection of a profitable skin site for biopsy, but in practice confusion over the basic diagnosis of leprosy is commoner. Browne $(1959,1964,1976)$ has drawn attention to the various ways in which onchocerciasis may mimic leprosy, or interfere with the diagnosis, and more recently Meyers et al. (1972) have described the close resemblance of hypopigmented skin lesions in streptocerciasis to those of leprosy in patients from Zaire; these authors conclude that biopsy may be the only way to distinguish the 2 diseases, especially in children.

Running through the diagnostic difficulties of onchocerciasis, streptocerciasis, granuloma multiforme, leprosy and many other tropical dermatoses, is the factor of altered skin pigmentation. In the case $\jmath_{\mathrm{f}}$ onchocerciasis, possible mechanisms have been reviewed in detail (Browne, 1954, 1960), though the definitive answer is still awaited, as it is also in streptocerciasis (Meyers et al., 1972), where neither the degree of pigmentary incontinence nor the numbers of MF are definitely related to hypopigmentation. In leprosy, where changes in skin pigmentation may be early and extremely important diagnostically (and of ten in the absence of any sensory changes or diminution of sweating), the mechanism of hypopigmentation is similarly obscure, though the theory has been repeatedly advanced (Prabhakaran et al., 1971, 1976) that dihydroxyphenylalanine (DOPA) may be an 
essential metabolite for the growth and multiplication of the leprosy bacillus, thus interfering with normal pigment production by melanocytes.

These derangements of pigmentation, together with the possibility that biting arthropods (Narayanan et al., 1972) or flies (Geater, 1975) may play a part in the transmission of leprosy suggest that there may be common ground of interest between leprosy and microfilariasis. This may be all the more worthy of investigation in view of the recent publication (Meyers and Connor, 1975) of a low frequency and reduced severity of Mazzotti reactions in patients with leprosy.

The value of slit-skin smears and skin-snips in the day-to-day handling of patients in the field cannot be over estimated and it is not our intention to belittle their importance. However, in further research on these 2 diseases, skin biopsies, properly taken and interpreted, may be invaluable. Furthermore, in the indiviclual patient, skin biopsy may be the best-and at times the only-way of establishing the correct diagnosis and classification of leprosy.

\section{Acknowledgements}

We are grateful to Dr S. G. Browne, Director of the Leprosy Study Centre, for permission to refer to clinical records and for much help in the writing of this paper, and to Dr D. J. Harman for allowing us to use the histopathological reports on which this paper is based. A. C. McDougall was supported by the British Leprosy Relief Association (LEPRA).

\section{References}

Browne, S. G.

Congo. M. D. Thesis, University of London.

Browne, S. G. (1959). La Gale Filarienne et la Lèpre. Ann. Soc. belg. Méd. trop. 39, 257.

Browne, S. G. (1960). Onchocercal depigmentation. Trans. R. Soc. trop. Med. Hyg. 54, 325.

Browne, S. G. (1964). In Leprosy in Theory and Practice,. p. 290. (Eds R. G. Cochrane and T. F. Davey.) John Wright and Sons Ltd, Bristol.

Browne, S. G. (1976). Onchocerciasis and the skin. Essay's in Tropical Dermatology. (Ed. J. Marshall.) Excerpta Medica, Amsterdam.

Buck, and Other Parasitic Diseases. WHO, Geneva, Switzerland.

Connor, D. H., Morrison,

Failing, F. C., Hale, L. N. and Lindquist, K. (1970). Onchocerciasis, onchocercal dermatitis, lymphadenitis and elephantiasis in the Ubangi Territory. Hum. Path. 1, 553.

Geater, J. G. (1975). 279.

Meyers, W. M. \& Connor, D. H. (1975). Onchocerciasis and streptocerciasis in patients with leprosy. Altered Mazzotti reactions. Trans. R. Soc. trop. Med. Hyg. 69, 524.

Meyers, W. M., Connor, D. H., Harman, L. E., Fleshman, K., Moris, R. and Neafie, R. C. (1972). Human streptocerciasis. Am. J. trop. Med. Hyg. 21, 528.

Narayanan, E., Shankara Manja, K., Bedi, B. M. S., Kirchheimer, W. F. and Balasubrahmanyan, M. (1972). Arthropod feeding experiments in lepromatous leprosy. Lepr. Rev. 43, 188.

Prabhakaran, K., Harris, E. B. and Kirchleimer, W. F. (1971). The interaction of Mycobacterium leprae and melanocytes in vitro. Cytobios. 4, 93.

Prabhakaran, K., Harris, in leprosy and occurrence of o-diphenoloxidase in Mycobacterium leprae. In Proceedings of the IX International Pigment Cell Conference. (Ed.

Ridley, D. S. and Jopling, W. H. (1966). Classification of leprosy according to immunity: a group system. Int. J. Lepr. 34, 255.

Wheeler, E. A., Hamilton, E. G. and Harman, D. J. (1965). An improved technique for the histopathological diagnosis and classification of leprosy. Lepr. Rev. 36, 37. 